\title{
High-resolution investigations of ripple structures formed by femtosecond laser irradiation of silicon
}

\author{
M. Schade $\cdot$ O. Varlamova $\cdot$ J. Reif $\cdot$ H. Blumtritt • \\ W. Erfurth $\cdot$ H. S. Leipner
}

Received: 31 August 2009/Revised: 27 October 2009 /Accepted: 4 November 2009/Published online: 13 December 2009

(C) The Author(s) 2009. This article is published with open access at Springerlink.com

\begin{abstract}
We report on the structural investigation of selforganized periodic microstructures (ripples) generated in $\mathrm{Si}$ (100) targets after multishot irradiation by approximately 100 -fs to 800 -nm laser pulses at intensities near the single shot ablation threshold. Inspection by surface sensitive microscopy, e.g., atomic force microscopy (AFM) or scanning electron microscopy (SEM), and conventional and high-resolution transmission electron microscopy reveal complex structural modifications upon interaction with the laser: even well outside the ablated area, the target surface exhibits fine ripple-like undulations, consisting of alternating crystalline and amorphous silicon. Inside the heavily modified area, amorphous silicon is found only in the valleys but not on the crests which, instead, consist of highly distorted crystalline phases, rich in defects.
\end{abstract}

Keywords Laser ablation · Ripple structure ·

Electron microscopy $\cdot$ Amorphous silicon

This contribution is based on a presentation given at the 15th Meeting on Solid State Analysis held from 12 to 16 July 2009 in Chemnitz, Germany.

M. Schade $(\bowtie) \cdot H$. S. Leipner

Interdisziplinäres Zentrum für Materialwissenschaften,

Martin-Luther-Universität Halle-Wittenberg,

06099 Halle, Germany

e-mail: martin.schade@cmat.uni-halle.de

O. Varlamova $\cdot$ J. Reif

LS Experimentalphysik II,

Brandenburgische Technische Universität Cottbus,

03046 Cottbus, Germany

H. Blumtritt $\cdot$ W. Erfurth

Max-Planck-Institut für Mikrostrukturphysik,

Weinberg 2 ,

06120 Halle, Germany

\section{Introduction}

Nanostructuring of silicon surface upon ultrashort pulse laser ablation has been extensively studied in recent years [1-18]. Surface sensitive investigations, e.g., scanning electron microscopy (SEM) or atomic force microscopy (AFM), reveal a large variety of patterns, depending on the incident dose of irradiation. At comparably low dose parallel regular modulations are observed, perpendicular to the laser polarization with a feature size significantly smaller than the wavelength. With increasing dose the pattern becomes more and more complex and coarser, even across the Gaussian beam profile [4, 9, 11, 13, 16, 17]. Interestingly, this variation does not simply follow the intensity profile but occurs in discrete steps, exhibiting the phenomenon of period doubling [17], typical for structure formation in nonlinear dynamics.

Electrostatic force microscopy (EFM) revealed a strong increase of the surface potential, following the morphological surface modulation of the nanostructures $[7,10]$. As one possible explanation, dopant segregation during structure formation was suggested.

Much less information is available about the crosssectional structure of the nano- and micropatterns. SEM experiments on cross-sectional cuts reveal narrow trenches of a very high aspect ratio between wider, almost flat crests $[6,12,15,18]$.

Initial transmission electron microscopy (TEM) analysis of such patterns generated by femtosecond laser irradiation show that part of the material was transformed from crystalline to amorphous $[18,19]$. Other experiments, not considering nanostructure formation, showed that irradiation by ultrafast laser pulses can result in both amorphization of crystalline material [20-23] and crystallization from the amorphous state [24]. Also, $\mu$-Raman spectroscopy of 


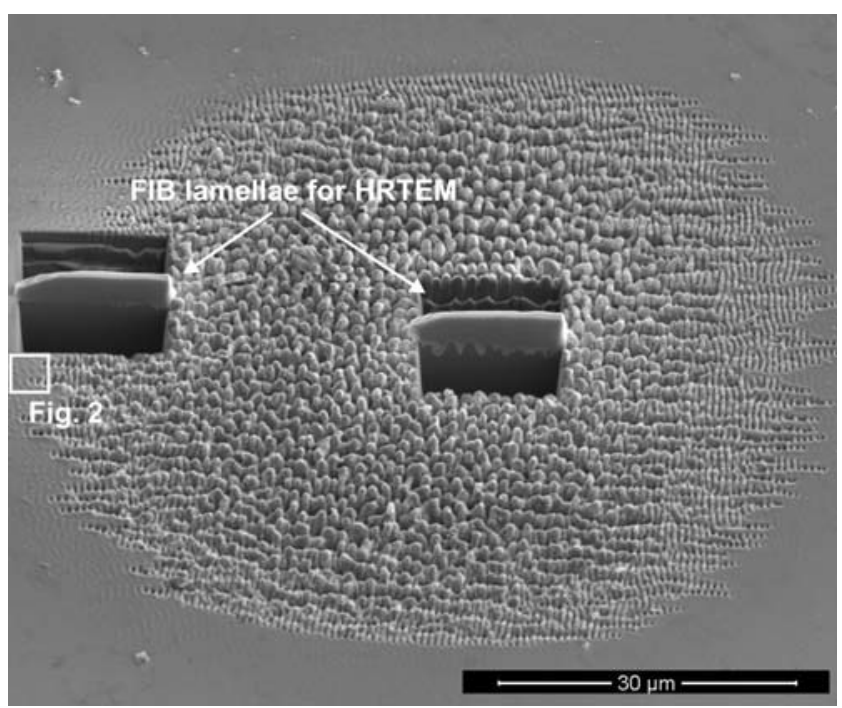

Fig. 1 SEM image of a typical ablation spot recorded before the lamellae were cut free during the FIB procedure

femtosecond laser ablated Si surface revealed the generation of both amorphous and more complicated (highpressure) phases of crystalline silicon $[4,5,25]$. For the origin of nanostructure formation upon femtosecond laser ablation, the following scenario was suggested $[8,9]$ : the rapid energy deposition as well as the surface decomposition during ablation result in a severe perturbation of the target crystalline system, far from thermal equilibrium. The subsequent rapid relaxation is much faster than any thermodynamic equilibrium process, giving rise to self-organized structure formation, which is not necessarily connected to the target structure before interaction. The detailed dynamics is still not fully clear. Therefore, the knowledge of the atomic fine structure in relation to the depth of the modified area should be an important key to a better understanding.

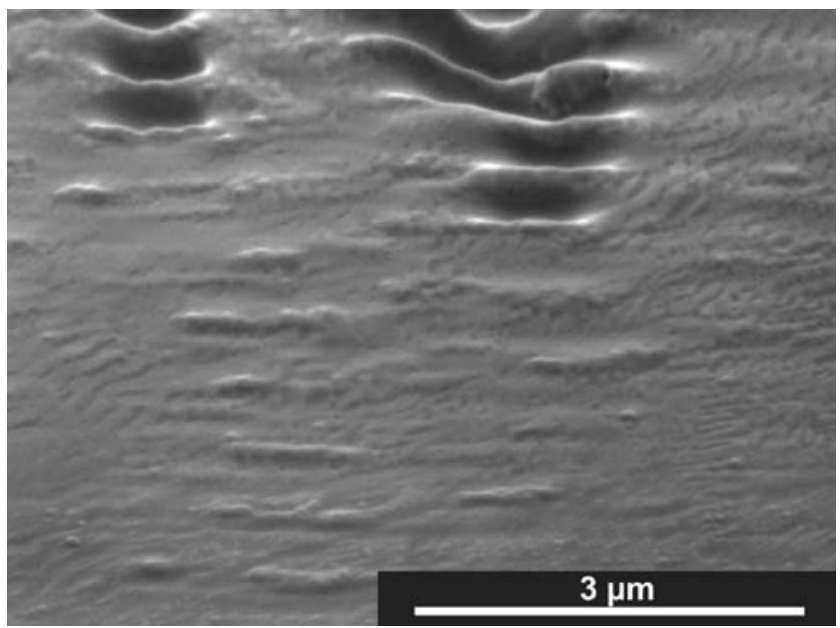

Fig. 2 Magnified SEM image of the rim of the crater (rotated $90^{\circ}$ relative to the box in Fig. 1). Surface undulations of different size appear around the ripples
The present paper is meant to extend the existing TEM studies on silicon subsurface structures upon the nonequilibrium phase transition process and patterning induced by femtosecond laser ablation from $\mathrm{Si}$, when exposing the target placed under ultrahigh vacuum to repetitive irradiation at fluences below the ablation threshold.

\section{Experimental}

Linearly polarized pulses from an amplified Ti:sapphire laser system $(800 \mathrm{~nm}, 100 \mathrm{fs}, 100-\mathrm{Hz}$ repetition rate) were applied to ablate and structure the surface of a commercial p-doped $\operatorname{Si}(100)$ wafer. The beam was focussed by a lens (focal length $300 \mathrm{~mm}$ ) onto spots of about $100-\mu \mathrm{m}$ diameter (FWHM) at about normal incidence onto the surface of the sample placed in an ultrahigh vacuum chamber (base pressure ca. $10^{-9}$ mbar). A CCD camera (Spiricon), placed in the focal plane, monitored the
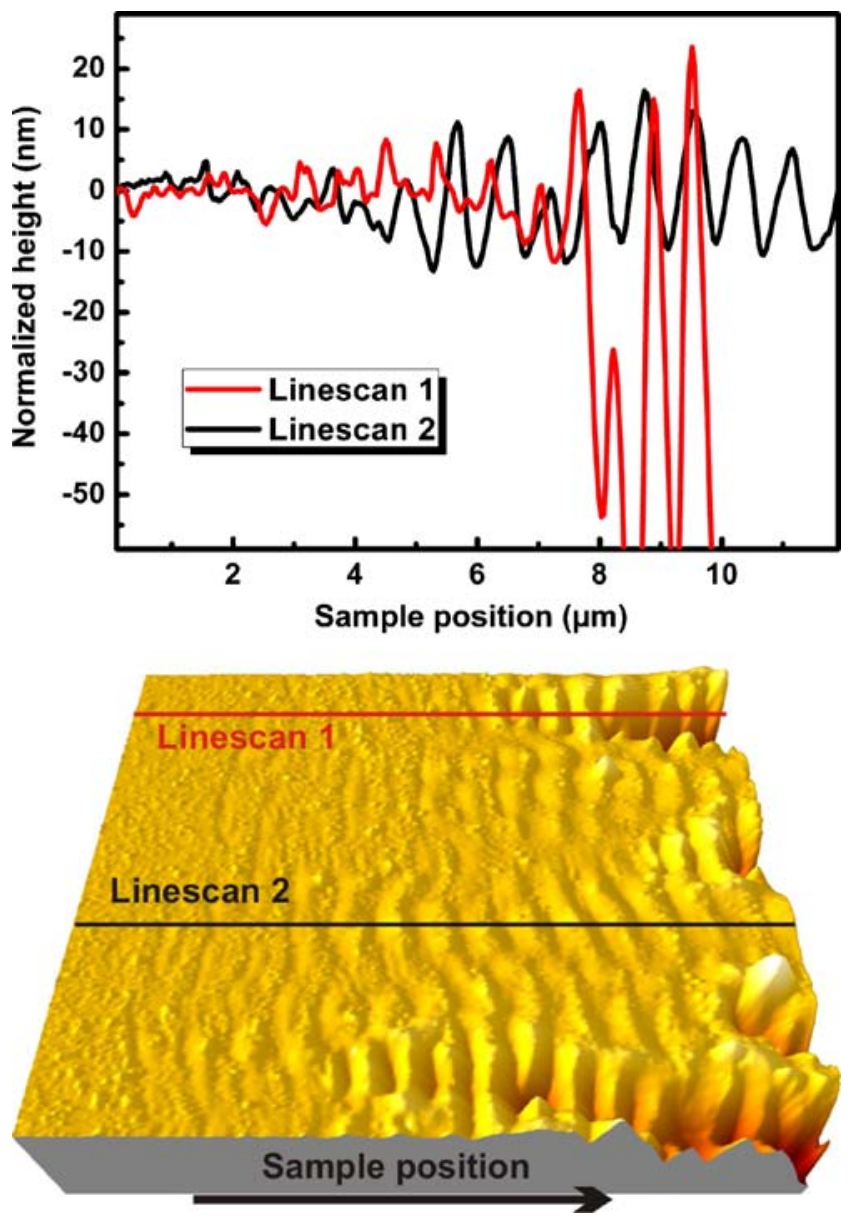

Fig. 3 The lower image shows a 3D plot of AFM data recorded at the rim of the crater, where the transition from ripples surrounded by deep depressions to surface undulations is visible. In the upper image associated depth profiles along the marked lines are illustrated. The profiles are normalized to the surface of the unmodified wafer 


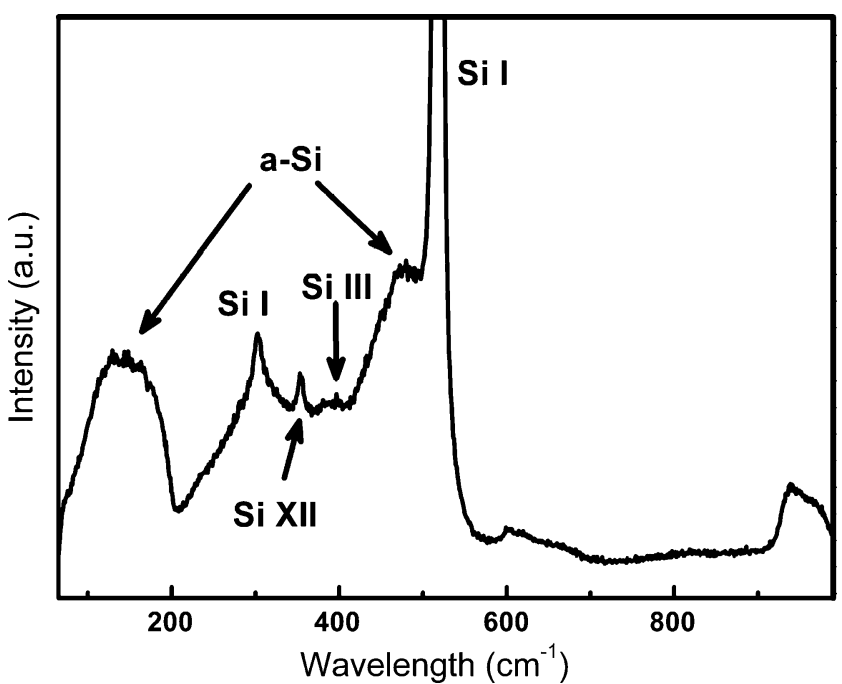

Fig. 4 Raman spectrum from the rim of the crater that exhibits the silicon phases Si I, Si III, and Si XII as well as amorphous silicon

approximately Gaussian intensity profile of the focussed laser beam. The experiments were carried out in multishot mode (n-on-1) with 1,000 pulses on one spot at an intensity of $1.1 \times 10^{12} \mathrm{~W} \mathrm{~cm}^{-2}$, clearly below the single shot ablation threshold for silicon $\left(I_{\mathrm{th}}=2 \times 10^{12} \mathrm{~W} \mathrm{~cm}^{-2}\right)$ [26]. The laser intensity was adjusted passively by polarization optics; the number of pulses was controlled precisely by an electromechanical shutter.

The resulting modified spots were inspected ex situ by scanning electron microscopy (SEM). In addition, the surface at the rim of the spots was investigated by atomic force microscopy (AFM) using a Pacific Nanotechnology Nano- $\mathrm{R}$ in tapping mode. The appearance of amorphous silicon at the spots was located by a Dilor LabRam confocal Raman microscope with 633-nm excitation wavelength from a $\mathrm{He}-\mathrm{Ne}$ laser, focussed onto a $1 \mu \mathrm{m}^{2}$ spot.

In order to investigate the in-depth cross section in the region of modified surface, two TEM cross section samples were prepared by using an FEI Nova Nanolab 600 DualBeam workstation, a focused ion beam (FIB)/scanning electron microscopy system. This technique enabled us to locate the specific site of interest. In practice, we first electron beam deposited a 100-nm-thick Pt layer to protect the surface of the samples from amorphization due to the high energy of the FIB. Then, a 3- $\mu \mathrm{m}$-thick Pt layer was ion beam deposited on the areas of interest with a size of $2 \mu \mathrm{m} \times 10 \mu \mathrm{m}$, each. Material was removed from both sides of the selected Ptprotected areas by successive thinning steps with decreasing FIB beam current until the lamellae were about $1-\mu \mathrm{m}$ thick, as can be seen in Fig. 1. Subsequently, the lamellae were cut free, transferred and fixed onto a specific TEM grid. The entire lamellae were thinned further to a thickness of around $200 \mathrm{~nm}$ using an ion beam of $30 \mathrm{kV}$, followed by cleaning steps with ion beams of $5 \mathrm{kV}$ and $2 \mathrm{kV}$, respectively, until a sample thickness of around $100 \mathrm{~nm}$ was achieved.

The electron transparent cross section samples were investigated by high-resolution transmission electron microscopy (HRTEM) using a JEM 4010 transmission electron microscope at an acceleration voltage of $400 \mathrm{kV}$.

\section{Results and discussion}

\section{Surface patterning}

An overview of the typical size and shape of an ablation spot is given in Fig. 1.

The modified area exhibits regular periodic surface structures (ripples). At the rim, narrow parallel ripples at high periodicity are observed, perpendicular to the electric field of the incident beam. Towards the spot center, i.e., higher laser intensity, the pattern becomes coarser and much more complex; however, the shape of the ripples also varies comparing the rim with the center. The apparent asymmetry of the spot can be attributed to a slight deviation from normal incidence. The resulting weaker intensity and wider slope at the horizontal parts of the rim are translated into a finer ripples structure, which is typical for lower irradiation dose [27].

Similar to observations at much higher intensity (and thus probable heat-affected zones) [23], weak surface modifications are observed as shown in Fig. 1, even outside the ablated area. A magnification of such an area is given in Fig. 2 which is a SEM image recorded at an electron voltage of $2 \mathrm{kV}$ in order to achieve a maximum morphological contrast. Whereas the modulation at the rim of the ablated area is characterized by very deep trenches between
Fig. 5 SEM images of the FIB prepared lamellae where both the ripples and the Pt cover layers are visible. The positions where the TEM images were recorded are indicated
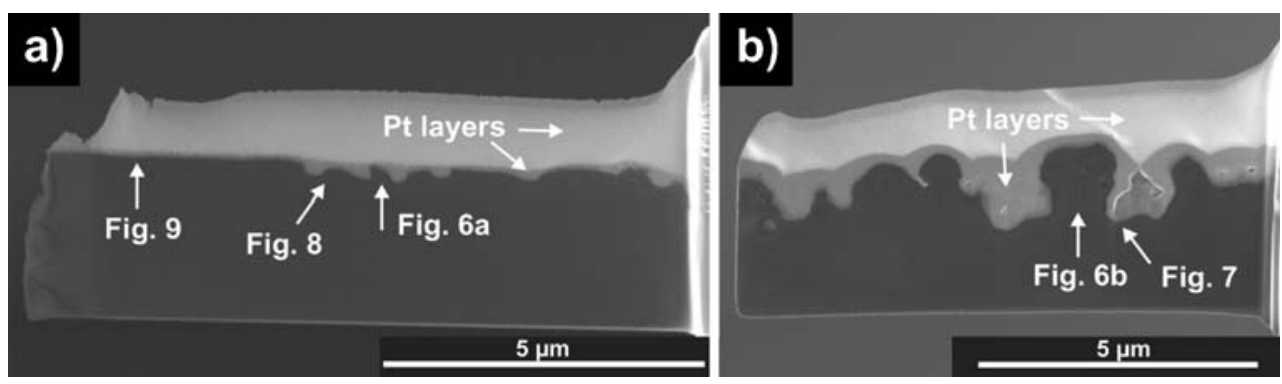
Fig. 6 BF-TEM micrographs of the bigger ripples in Fig. 5. The crystal structure inside the ripples is distorted heavily. The depressions around the ripples are filled with amorphous silicon
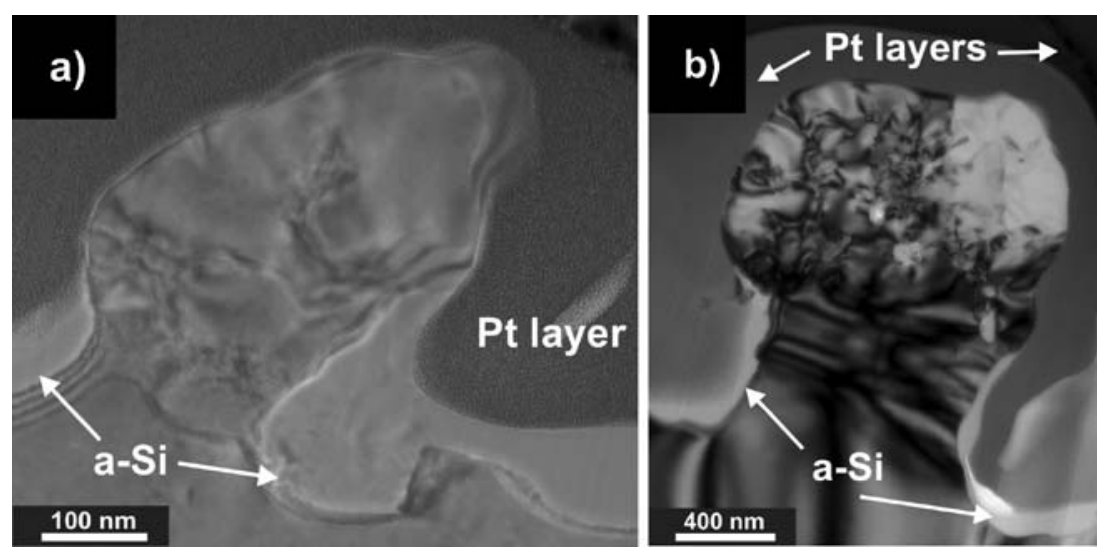

the ripples, further out the ripples appear as slight crests above the unperturbed surface, but preserving the regularity. Ripples and crests are surrounded by tiny surface undulations exhibiting various orientations: several undulations are orientated parallel to the ripples or crests, respectively, whereas others are perpendicular or at a slight angle.

Atomic force microscopy investigations yield further information about the size and the range of the surface undulations. Figure 3 shows a 3D plot of AFM data scanned over a $12 \mu \mathrm{m} \times 12 \mu \mathrm{m}$ region at the rim of the crater. The 3D plot confirms the high aspect ratio of the trenches in the ablation area. The transition to crests and surface undulations is demonstrated clearly in Fig. 3. The spacing between adjacent ripples, and hence their periodicity, remains nearly constant and is continued for the crests further out. At two regions (marked linescan 1 and linescan 2 ), depth profiles were extracted from the AFM data and are also plotted in Fig. 3. The profiles are normalized to the

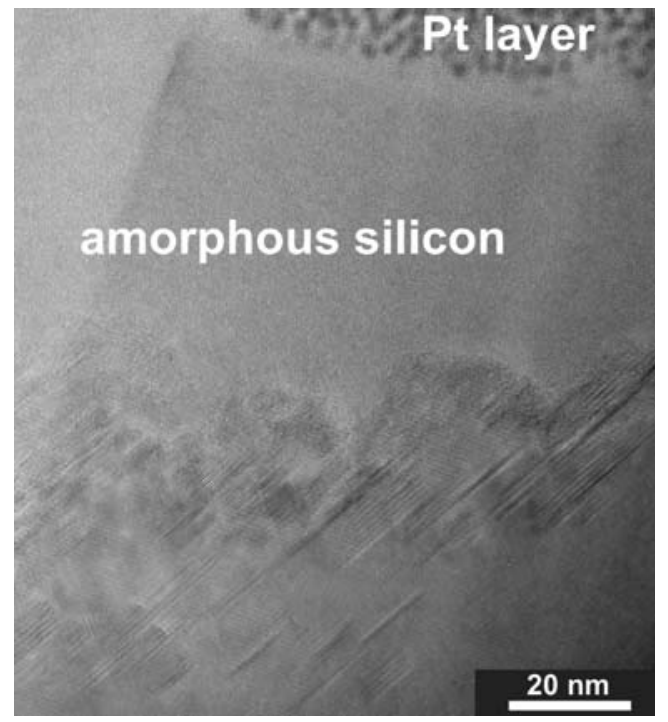

Fig. 7 Magnification of the shoulder of the ripple in Fig. 6b. Various defects appear in the crystalline silicon that is capped by amorphous silicon wafer surface well outside the affected area. Interestingly, the profiles indicate that part of the ripples structure rises above the initial surface, whereas the valleys in between appear depressed. This confirms the hypothesis that the structures originates from self-organization from a surface instability $[8,9]$ and not from mere ablation or phase transformation. Comparing the two profiles, differences in height and width of the crests as well as the lateral shift are visible. A correlation between these features and the distance to the center of the crater could not be observed.

The irradiated crystalline silicon (Si I) undergoes complex transformations during ablation and structure formation. Therefore, the structured surface was analyzed additionally by measuring Raman spectra at the inside of the crater and its rim. Figure 4 shows a representative Raman spectrum: at the center of the crater and its rim the bcc and rhombohedral phases (Si III, Si XII) appear in addition to amorphous silicon (a-Si) [4, 5]. Raman spectra from the surface undulations exhibit no contributions from silicon polymorphs, but weak contributions of amorphous silicon are still present. It should be noted here that such silicon polymorphs are frequently considered to result from high pressure acting on the surface [28]. Indeed, a coarse estimate, taking into account the high kinetic energy of desorbed particles of a few tens of electron volts [4], indicates the possibility of a recoil pressure of some tens of megapascals.



Fig. 8 BF-TEM micrograph of a ripple showing a special feature: amorphous silicon appears in a hollow at its top 


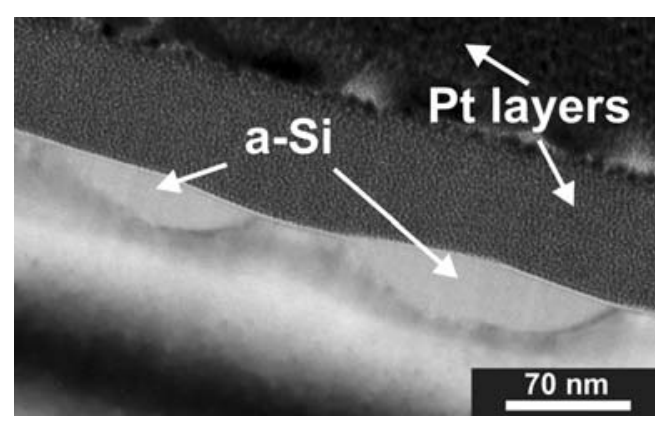

Fig. 9 BF-TEM pattern showing two hollows filled with a-Si inside the crystalline silicon. The interface between silicon and the Pt layer is uneven

TEM investigations of cross section samples

SEM images of the cross section samples from the positions indicated in Fig. 1 are illustrated in Fig. 5; Fig. 5a represents the lamella from the rim and Fig. 5b the lamella from the center. The Pt cover layers and the ripple structures are well distinguishable through their changing contrast. Comparing the lamellae, a strong difference in size, shape, and regularity of both the ripples and the trenches is ascertained. Bright field (BF) TEM micrographs of individual ripples (cf. Fig. 5) from both lamellae are illustrated in Fig. 6. The ripple in Fig. 6a mainly consists of a heavily distorted region that merges into crystalline silicon at the ripple's base. The ripple in Fig. 6b exhibits a similar behavior. However, the top region is much strongly distorted. In addition, the dark lines at the base of the ripple reveal restraints that are induced partially by the low thickness of the lamella.

Both micrographs reveal regions with different contrast at the shoulders of the ripples. Hence, HRTEM micrographs of these regions were acquired to obain further information about their structure. Figure 7 shows a representative micrograph, recorded at the position indicated in Fig. 5b, which can be divided into three parts: the lower part consists of crystalline silicon where stacking faults and microtwins appear. The upper part shows the Pt cover layer. Between these two parts the image reveals an approximately $60-\mathrm{nm}$-thick amorphous layer which should be amorphous silicon, since the experiments occurred under ultrahigh vacuum conditions and the formation of an oxide should be greatly reduced. An amorphization of this layer caused by $\mathrm{Ga}^{+}$ion irradiation during sample preparation can be excluded because of the low energy deposition of the first Pt cover layer. The interface between crystalline silicon and amorphous silicon appears roughened, whereas a transition region a few nanometer thick, where both phases coexist, could be observed. Figure 8 shows a ripple located at the rim of the crater. The shoulders of the ripple are covered, again, with amorphous silicon. A special feature of this ripple is the presence of a hollow, filled with amorphous silicon at its top. This might indicate that, in fact, there are two ripples. On the other hand, this structure could demonstrate an intermediate state during the formation of ripples. BF-TEM micrographs around the last trench in Fig. 5a show that at the end of this trench crystalline silicon regions continue the "crest" series, whereas the "trench" series is replaced by amorphous regions. This behavior is visualized in Fig. 9 in a BF-TEM micrograph recorded far away from the last trench appearing in the lamella (see Fig. 5a). The image exhibits two hollows in the crystalline silicon: HRTEM micrographs (not shown) verify the assumed filling with amorphous silicon as well as the transition region between crystalline silicon and amorphous silicon, already observed at the shoulders of the ripples. In this area, however, dislocations and stacking faults could be detected in the crystalline silicon only sporadically between the hollows.

The interface between the crystalline or amorphous silicon and the Pt cover layer consists of an approximately 4-nm-thick amorphous layer which appears in Fig. 9 as a narrow white line. By applying electron energy loss spectroscopy (EELS), the investigation of the fine structure of the silicon $\mathrm{L}_{2,3}$ edge reveals that this amorphous layer consists of $\mathrm{SiO}_{2}$ (not shown). In addition, EELS inves-
Fig. 10 HRTEM micrographs of the distorted regions inside the ripples in a Fig. $6 \mathrm{~b}$ and $\mathbf{b}$ Fig. 6a. The insets show diffraction patterns of the distorted regions. A superstructure typical for an area of equally spaced microtwins becomes evident
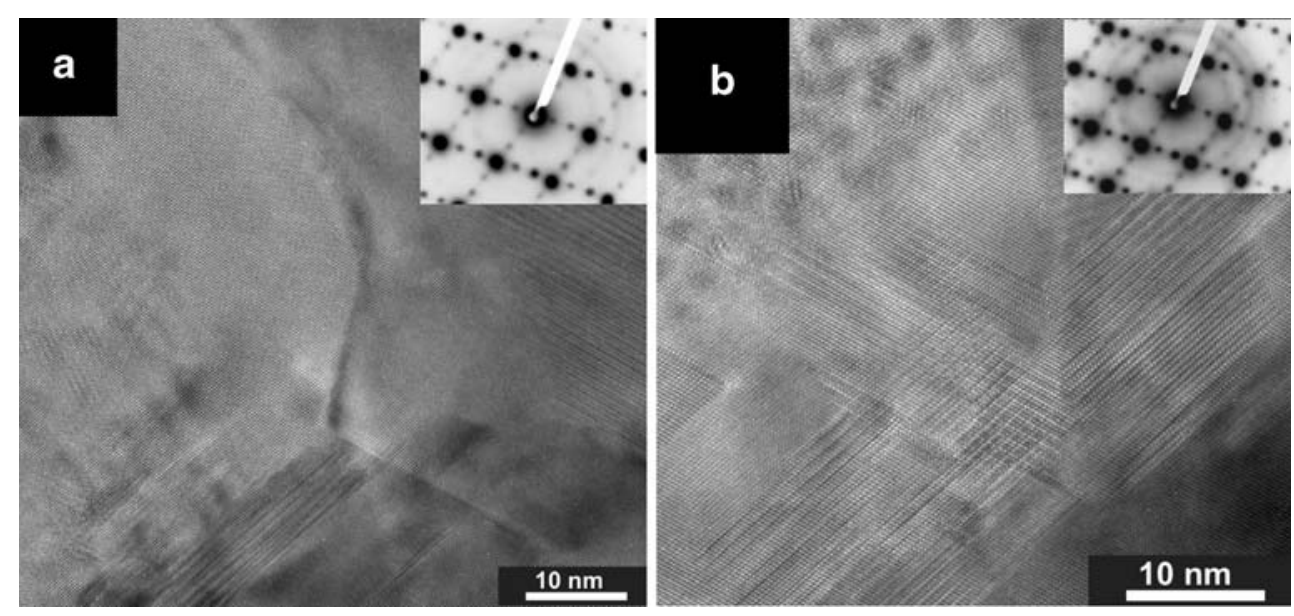
tigations of the amorphous material in the hollows and at the shoulders of the ripples could verify the presence of a$\mathrm{Si}$ due to characteristic differences in the respective silicon $\mathrm{L}_{2,3}$ edges. Since the $\mathrm{SiO}_{2}$ layer is slightly thicker than the native $\mathrm{SiO}_{\mathrm{x}}$ layer of the original silicon wafer, an influence of either the $\mathrm{Ga}^{+}$ion irradiation during sample preparation or the irradiation by femtosecond laser pulses onto the thickness of the $\mathrm{SiO}_{2}$ layer cannot be excluded.

Furthermore, this interface is uneven: it is shifted towards the Pt layer at the positions of the amorphous silicon. We suggest that these hollows filled with amorphous silicon are identical to the surface undulations detected by SEM and AFM, respectively. This conclusion could also explain the contributions of amorphous silicon in the Raman spectra recorded from the surface undulations.

Another point of great interest concerns the structures inside the heavily distorted regions and at the surface of the ripples. Figure 10a shows an HRTEM micrograph of the distorted region in Fig. 6b; Fig. 10b illustrates a part of the distorted region in Fig. 6a. Both micrographs exhibit the same structures: twin boundaries and stacking faults accompanied by dislocations occur primarily. In addition, grains with different lattice orientations were observed by applying HRTEM. A definite assignment of these grains to the silicon polymorphs observed in the Raman spectra failed, however. A possible disappearance of the silicon polymorphs due to heating during the FIB preparation could not be excluded. The surface of the top part of a ripple is visible in Fig. 10b where the incipient Pt layer appears as dark spots in the upper left part of the image. Similar to this image all other HRTEM micrographs recorded from the surface at the distorted regions exhibit the same behavior: no amorphous silicon or silicon oxide layer could be detected at the interface between ripples and the Pt layer. This conclusion is supported by the BF-TEM images in Fig. 6 where an appropriate contrast appears only at the shoulders of the ripples.

\section{Conclusion}

We described the characterization of surface structures formed by femtosecond laser irradiation of $\mathrm{Si}(100)$. SEM images and AFM patterns of the modified area reveal surface undulations with variable orientations beyond the ripples. Bigger surface undulations also form periodic chain-like structures, whereas adjacent chains are shifted against each other. An interaction between smallest surface undulations and the ripples is assumed due to their arrangement. Raman spectra in combination with HRTEM micrographs show that these surface undulations consist of amorphous silicon. HRTEM micrographs show ripple surfaces free from amorphous silicon, which is only found at the shoulders of the ripples and at the bottom of the trenches. Since SEM and AFM patterns exhibit a high regularity in the structures across the transition from ripples to surface undulations the role of the amorphization of crystalline silicon during the formation process of ripple structures has to be the subject of further research. Polymorphs of crystalline silicon appearing in Raman spectra recorded from the ripple structures $[4,5]$ are confirmed. BF-TEM images reveal huge distorted crystalline regions inside the ripples: corresponding HRTEM micrographs exhibit primarily twin boundaries and stacking faults accompanied by dislocations. These lattice defects appear beneath the bottom of the trenches, too.

Acknowledgement The authors thank the Max Planck Institute of Microstructure Physics, Halle, for use of the transmission electron microscope JEOL 4010.

Open Access This article is distributed under the terms of the Creative Commons Attribution Noncommercial License which permits any noncommercial use, distribution, and reproduction in any medium, provided the original author(s) and source are credited.

\section{References}

1. Her T-H, Finlay RJ, Wu C, Deliwata S, Mazur E (1998) Appl Phys Lett 73:1673

2. Krüger J, Kautek W (1999) Laser Phys 9:30

3. Borowiec A, Haugen HK (2003) Appl Phys Lett 82:4462

4. Costache F, Kouteva-Arguirova S, Reif J (2004) Appl Phys A 79:1429-1432

5. Bonse J, Brzezinka K-W, Meixner AJ (2004) Appl Surf Sci 221:215

6. Coyne E, Magee JP, Mannion P, O'Connor GM, Glynn TJ (2005) Appl Phys A 81(2):371-378

7. Reif J, Ratzke M, Varlamova O, Costache F (2006) Mat Sci Eng B 134:114

8. Varlamova O, Costache F, Reif J, Bestehorn M (2006) Appl Surf Sci 252:4702

9. Reif J, Costache F, Bestehorn M (2006) Self-organized surface nano-structuring by femtosecond laser processing; chapter 9 . In: Perriere J, Millon E, Fogarassy E (eds) Recent advances in laser processing of materials. Elsevier, Amsterdam, p 275

10. Varlamova O, Costache F, Ratzke M, Reif J (2007) Appl Surf Sci 253:7932

11. Tomita T, Kinoshita K, Matsuo S, Hashimoto S (2007) Appl Phys Lett 90:153115

12. Couillard M, Borowiec A, Haugen HK, Preston JS, Griswold EM, Botton GA (2007) J Appl Phys 101(3):033519

13. Okada T, Kawahara H, Ishida Y, Kumai R, Tomita T, Matsuo S, Hashimoto S, Kawamoto M, Makita Y, Yamaguchi M (2008) Appl Phys A 92(3):665-668

14. Miyaji G, Miyazaki K (2008) Opt Express 16:16265

15. Crawford THR, Yamanaka J, Botton GA, Haugen HK (2008) J Appl Phys 103(5):053104

16. Reif J, Varlamova O, Costache F (2009) Appl Phys A 92:1019

17. Reif J, Costache F, Jia G, Varlamova O, Ratzke M (2009) Status Solidi C 6:681 
18. Tomita T, Kumai R, Matsuo S, Hashimoto S, Yamaguchi M (2009) Appl Phys A 97:271-276

19. Höche TH, Ruthe D, Frost F, Petsch T (2003) Proc ICALEO 2003 (Laser Inst Am ) A33

20. Höche Th, Ruthe D, Petsch T (2004) Appl Phys A 79:961

21. Jia J, Li M, Thompson CV (2004) Appl Phys Lett 84:3205

22. Izawa Y, Izawa Y, Setsuhara Y, Hashida M, Fujita M, Sasaki R, Nagai H, Yoshida M (2007) Appl Phys Lett 90(4): 044107+

23. Picard YN, Yalisove SM (2008) Appl Phys Lett 92:014102
24. Zhang G, Gu D, Gan F (2004) Solid State Commun 131:211

25. George A (1999) High pressure phases of c-Si. In: Hull R (ed) Properties of crystalline silicon. INSPEC 104

26. Bonse J, Baudach S, Krüger J, Kautek W, Lenzner M (2002) Appl Phys A 74:19-25

27. Reif J, Varlamova O, Costache F, Eckert S (2007) Proc SPIE 6606:66060

28. Kouteva Arguirova S, Orlov V, Seifert W, Richter H, Reif J (2003) Solid State Phenomena 95-96:513 